Dear Editor,

\title{
Characterizing the negative binomial distribution
}

Recently the authors, Cacoullos and Papageorgiou (1982) and Papageorgiou (1982), have been doing some work on characterizing a discrete random variable $Y$ which has a mixture representation in terms of another discrete random variable $X(t)$, where the parameter $t$ plays the role of the mixing variable. Of special interest is the case of a Poisson process $\{X(t), t>0\}$ where the time parameter $t$ may be regarded as a random variable. The probability function $p(y)$ of $Y$, in such a case, is given by the Poisson mixture

$$
p(y)=\int_{0}^{\infty} e^{-t} t^{y} / y ! d F(t),
$$

where $F$ denotes the distribution function of $t$.

Engel and Zijlstra (1980), 'investigating a practical problem... met a characterization, not mentioned in these surveys'; they refer to surveys in characterizations of the gamma distribution. Assuming that delivery orders for some product follow a Poisson process, they show, in effect, that the distribution of (the number of orders) $Y$ (during a lead time) is negative binomial if and only if (the lead time) $t$ has a gamma distribution.

The purpose of this note is twofold. First, to point out that the preceding characterization of the negative binomial distribution follows from the identifiability of Poisson mixtures, that is, the one-to-one correspondence between $p(y)$ and $F(t)$, as shown by Teicher (1961); that a gamma $F$ yields a negative binomial $Y$ is a rather old and well-known result. Second, to indicate briefly a related characterization, which may be of some practical interest as well.

Consider the regression $m(y)$ of the time variable $t$ on $Y$ and suppose this is linear, namely, $m(y)=\alpha+\beta y, \alpha>0,0<\beta<1$. Then $t$ follows a gamma distribution (with scale parameter $\lambda=\beta /(1-\beta)$ and shape parameter $k=\alpha / \beta$ ) and $Y$ follows the negative binomial distribution

$$
p(y)=\left(\begin{array}{c}
k+y-1 \\
y
\end{array}\right) \lambda^{y}(1+\lambda)^{-(k+y)} .
$$

This is a special case of a more general result: if $Y$ is a Poisson mixture, then the regression function $m(y)$ of $t$ on $Y$ determines uniquely both the distribution of $t$ and $Y$. To show this, it is enough to establish that

$$
m(y)=(y+1) p(y+1) / p(y),
$$

which is a first-order difference equation, with a unique solution $p(y)$. 
Another simple application of the general result yields a characterization of the 'Poisson-Lindley' distribution (cf. Sankaran (1970)): if for some $\theta>0$,

$$
m(y)=(y+1)(\theta+3+y) /(\theta+1)(\theta+2+y),
$$

then $t$ and $Y$ have p.f.'s given by

$$
f(t)=\frac{\theta^{2}}{\theta+1}(t+1) e^{-\theta t}(t>0), \quad p(y)=\theta^{2} \frac{y+\theta+2}{(\theta+1)^{y+3}}, \quad y=0,1, \cdots .
$$

It should be mentioned that similar results hold for mixtures of the negative binomial with respect to the index parameter $k$. Now the corresponding difference equation becomes

$$
m(y)=\frac{\lambda+1}{\lambda}(y+1) \frac{p(y+1)}{p(y)}-y .
$$

Detailed results on characterizations of mixtures with $X(t)$ and $t$ both discrete, univariate or multivariate, will appear shortly, as stated earlier.

Statistical Unit, Athens

\author{
Yours sincerely, \\ Theophilos Cacoullos \\ H. PAPAGEORgIOU
}

\title{
References
}

Cacoullos, T. and Papageorgiou, H. (1982) Characterizations of discrete distributions by a conditional distribution and a regression function. Ann. Inst. Statist. Math.

ENGEL, J. AND ZIJLSTRA, M. (1980) A characterization of the gamma distribution by the negative binomial distribution. J. Appl. Prob. 17, 1138-1144.

PAPAgeORgiou, H. (1982) On characterizing some bivariate discrete distributions. Austral. J. Statist.

Sankaran, M. (1970) The discrete Poisson-Lindley distribution. Biometrics 26, 145-149.

Teicher, H. (1961) Identifiability of mixtures. Ann. Math. Statist. 32, 244-248. 